\title{
The cancer disaster: psychology of informal caregivers
}

\begin{abstract}
Cancers surface among one of the leading causes of mortality and morbidity worldwide. With umpteen cases being revealed every year, this killer disease seems to be tightening its grip in India too. Experts say that the incidence of cancer is expected to rise five-fold by the year 2025. As the cases of cancer are increasing, so is the need for the informal caregiving that is rising manifold. Caring for a beloved member of the family or a friend who is suffering from a deadly disease is in itself a big challenge. Taking on new responsibilities on one hand and worrying about the future can be very exhausting at the very least and might quickly lead to burnout. The major concern here is that when you are busy taking care of a person with such a care demanding disease, who is taking care of you? The informal caregivers of cancer patients undergo through fear, emotional burden, anxiety, depression and a lot of physical problems too. Their needs need to be identified and they need support in order to manage their caregiving role without harming their own health and psychological wellbeing. Significant amount of research work has shown that the informal caregivers suffer from the physical, mental and emotional problems that emanate from the complex caregiving situations in chronic diseases like cancer. Mostly the psychological health of the informal caregivers of cancer patients are put at great risk. The purpose of the present study is to review the literature on the psychological health and wellbeing of the informal caregivers of cancer patients along with the role of cultural factors in caregiving in India. Various online journals, PubMed journals, web searches, PsycInfo databases and online publications of various organisations related to the informal caregiving of cancer patients were accessed for the study. A number of review articles, studies and meta-analysis of studies pinpointing on the psychological health of the informal caregivers of cancer patients were reviewed. A number of studies indicated that anxiety and depression are the most common effects of caregiving while some studies also came out with the positive consequences associated with the role of caregiving like enhanced self-esteem and positive utility derived from caregiving. Rigorous research is required in this field so that the programmes and policymakers can work a great deal to come up with interventions for the betterment of the psychological health of the informal caregivers of cancer patients.
\end{abstract}

Keywords: burden, culture, depression, informal caregivers, psychological health, social support
Volume 7 Issue I - 2017

\author{
Purnima Awasthi,' Kuhu² \\ 'Associate Professor, Department of Psychology, Banaras Hindu \\ University, India \\ ${ }^{2}$ Research Scholar, Department of Psychology, Banaras Hindu \\ University, India
}

\begin{abstract}
Correspondence: Purnima Awasthi,Associate Professor, Department of Psychology, Banaras Hindu University, Varanasi 221 005, India, Email awasthip9@gmail.com
\end{abstract}

Received: November 25, 2016 | Published: January 12, 2017

\section{Introduction}

Department of Public Health ${ }^{1}$ defines informal caregiving as the help and support family members and friends provide daily to individuals who are temporarily or permanently unable to function independently. Caregiving involves a range of activities such as assisting with personal hygiene, helping with medication, doctors' visits, managing finances, acting as a patient advocate and providing emotional support. Informal caregivers often have a long term commitment to the persons they are helping; they usually are not paid for their efforts or trained for the tasks that are performed. The problems of caregivers can increase health and social costs: as some authors point out, caregivers are 'hidden patients' and as a consequence, are underestimated and undertreated. ${ }^{2}$ Furthermore, such caregiver problems increase the difficulties inherent in the relations between patient and caregiver. ${ }^{3}$

The latest figures published in the British medical journal, The Lancet show that more than 575,000 people die of cancer and more than 1.5 million people are diagnosed with cancer per year in the US. According to the World Health Organization ${ }^{4}$ the numbers of new cancer cases is expected to rise by about 70 per cent over the next 20years (Crosta 2015). Around 700,000 people are dying from cancer in India annually projected to rise to around 1.2 million deaths in 2035. ${ }^{5}$
Cancer has a ripple effect on the family members and informal caregivers of the cancer patients. It is impact has been compared to the dropping of a stone in the pond. Once you drop a stone in the pond, it causes some changes. Similarly once a family member is diagnosed with cancer, the family members are directly confronted with the illness experience. The members of the family provide the context of adjustment in which the person diagnosed with cancer responds to her/ his disease. Although the disease dwells in the body of the patient only, but it causes a huge psychological effect on the informal caregivers of the patient i.e. the family members or friends. Historically caregiving has not been a topic of much concern and focus for the research. With the decline in healthcare resources and rising healthcare costs, informal caregiving is gathering attention by the researchers.

\section{Negative psychological effects on informal caregivers of cancer patients}

Cotrim and Pereira ${ }^{6}$ identified and assessed the impact of colorectal cancer on colorectal cancer patients and their families. A cross sectional study with a correlational design was done on 96 informal caregivers. The measures used for the caregivers included the Beck depression inventory, state trait anxiety inventory and the burden assessment scale. It was found that the colorectal cancer has a major impact at the level of psychological morbidity. It also showed that 
high levels of burden related to the care of patients with cancer. This would probably lead to more stress resulting into depression. Also significant levels of differences were found between the depression and state anxiety of the stoma and the non- stoma patients.

Pinquart \& Sorensen ${ }^{7}$ conducted a meta-analysis on the differences between the caregivers and the non-caregivers in psychological and physical health. The inclusion criteria included a sample of informal caregivers of older adults compared to a sample of non-caregivers with regard to perceived stress, depression, general subjective wellbeing, physical health or self-efficacy and the differences between the caregivers and the non-caregivers can be converted into standard deviation units. Perceived stress was measured with Perceived Stress scale, depression with Hamiltan depression rating scale, Beck depression inventory and other scales like positive affect scales and self- efficacy scales were also used. Two common groups of statistical procedures were used in the Meta-analysis- fixed and random effect models. The effect of caregiver status was tested on the psychological and physical health. It was found that the caregivers had higher levels of stress and depression and lower levels of subjective wellbeing, physical health and self-efficacy than the non-caregivers. On a whole the meta-analysis suggested that the caregivers fare worse than the non-caregivers as they are more stressed, depressed and have lower levels of subjective wellbeing and self-efficacy than the noncaregivers.

Nijboer et al., ${ }^{8}$ did a study in Netherlands on the determinants of caregiving experiences and mental health of partners of cancer patients. This was a longitudinal study. The caregiver's experiences were assessed by Caregiver Reaction Assessment Scale which consisted of four negative sub scales and one positive sub scale. The mental health of the caregivers was assessed in terms of depression and quality of life. The level of depression of caregiverwas measured by Centre for Epidemiological Studies-Depression (CES-D). The caregiver's initial quality of life was measured with a one item linear visual analogue self-assessment scale. The results indicated that although caregiving may lead to depression, especially in those experiencing loss of physical strength, caregivers may sustain their quality of life by deriving self-esteem from caregiving.

Grunfeld et al., ${ }^{9}$ did a longitudinal study of breast cancer patients and their principal caregivers. Out of 89 caregivers, around half were males. The psychological distress was measured by the Hospital Anxiety and Depression scale. The other measures used were Zarit burden inventory, satisfaction with care was measured using FAMCARE instrument and Medical Outcome Study social Support survey was used to measure the patient's social support. The psychological impact of caregiving experience showed that the similar proportions of caregivers were anxious at the start of the terminal period as at the start of the palliative period. ( 39 per cent v. 9 per cent), $\mathrm{p}=0.18$ ). More of these caregivers were found to be depressed ( 30 per cent v. 9 per cent, $p=0.02$ ) at the start of the terminal period than at the start of the palliative period. However caregiver burden was found to be the most important predictor of anxiety and depression.

A review of research on the impact of caregiving on the psychological well being of family caregivers and cancer patients was done by Northhouse et al., ${ }^{10}$ This review of literature confirmed that cancer can affect the quality of life of family caregivers in many ways, but particularly in the psychological domain. It was also indicated that the patient and the caregiver distress is evident in each phase of illness but is found to be greater in some phases like diagnosis phase and the survivorship phase.
Goldzweig et al., ${ }^{11}$ did a study on 216 partners (96 men, 120 women) who were primary caregivers of cancer patient aged $60+$ and compared them with 76 partners of healthy people aged $60+$ and diagnosed with any terminal illness. Partners were interviewed by the psychologists. Each cohort was divided into two age groupsYounger (age60-70) and Older (age 70+). The measures utilised for the study were background variables, Brief symptom inventory, Brief cope and Social Support. A two-way MANOVA (Group $\times$ Patient's Age) compared levels of psychological distress reported by older and younger participants within the research and control groups. Levels of distress measured among the research group were significantly higher and about triple the distress measures reported by the control group participants. Moreover, within the research group, but not within the control group, caregivers to older patients with cancer reported distress levels doubling the distress levels reported by caregivers to younger cancer patient's interaction effect. Thus caregivers to cancer patients reported high levels of distress, low levels of social support and low levels of coping abilities which are negatively correlated to distress. Also increased patient age was found to accentuate these processes. However further researches need to focus on the variables that make the patients age an important factor in contributing to caregivers illness.

Braun et al., ${ }^{12}$ did a study to assess psychological distress among advanced cancer patients and their spouse caregivers, while examining the contribution of caregiving burden and relational variables (attachment orientation and marital satisfaction) to depressive symptoms in the spouse caregivers. The sample $(\mathrm{n}=101)$ spouse caregivers were recruited for the study. The measures used were Beck Depression Inventory (BDI-II), Caregiving Burden Scale, and Experiences in Close Relationships Scale and ENRICH Marital Satisfaction Scale. The data was analysed using the SPSS. Hierarchical regression was used to examine the main predictions. Hierarchical regression was used to determine to what extent subjective caregiving burden, attachment scores and marital satisfaction contribute to the prediction of spouse caregiver's depression, beyond the contribution of objective caregiving burden.

Overall, this model explained a total of $37 \%$ of variance of spouse caregiver's depression scores, with subjective caregiving burden, caregiver's anxiety and avoidance attachment orientations and marital satisfaction remaining as the significant predictors. The study is unique in providing information about the contribution of relational variables about the caregiver's depression. Higher caregiver's attachment anxiety and avoidance were associated with more depression. Overall the findings from this study suggested high prevalence of depression in spouse caregivers of patients with advanced cancer.

Song et al., ${ }^{13}$ did a study in Korea to study the quality of life and mental health in family caregivers of patients with terminal cancer. The family caregivers of terminal cancer patients who received palliative care services in Korea were surveyed. Control individuals were selected from the participants in the first round of fourth Korea National Health and Nutrition Examination Survey and were matched with family caregivers using the propensity score method to optimize the comparative analysis . The Emotional Quotient (EQ-5D) was used to assess health related quality of life using an index score and a visual analog scale. The Caregiver Assessment Scale was also used. The results revealed that the health related quality of life is significantly lower than that of the general population. Also the caregiver's level of anxiety and depression were found to be significantly different from that of the general population. 


\section{Positive psychological effects on the informal caregivers of cancer patients}

Brouwer et al., ${ }^{15}$ found out that the process of caregiving does not always lead to negative psychological effects on the caregivers but also has a positive effect on their psychology. This study was based on the fact that utility is not always derived from the outcome but also from the process involved in reaching to the outcome. The empirical evidence of process utility was presented on the basis of a large sample of 950 Dutch caregivers. The process utility was measured as the difference in happiness between the current situation in which the patient is cared for by the caregiver and the hypothetical situation that someone else takes over the care tasks, all other things equal. The caregivers were given questionnaires to probe into the background information, the objective burden was assessed and the subjective burden of caregiving was assessed using the Caregiver Strain Index and a Self-Rated Burden Scale.

The process utility was measured by comparing the two situations mentioned above in terms of happiness. The results indicated that the influence of process utility on total happiness can be substantial. It was found that some caregivers would lose a major part of their happiness if asked to give up their caregiving role. The process utility was affected by the caregiver characteristics and the subjective burden of caregiving too. However it seems as though focussing only on the negative side of the informal care does not do justice with the value of informal care. So on an average the happiness would decline when the caregiving tasks were handed to someone else.

A unique finding was derived in the study by Nijboer et al., ${ }^{8}$ in Netherlands on the determinants of caregiving experiences and mental health of partners of cancer patients. The findings suggested that the caregivers with a high level of education derived low selfesteem during the course of caregiving than with the caregivers with a low level of education. Thus for the caregivers with low level of education the task of caregiving would lead to the positive outcome of a higher level of self-esteem.

\section{Role of cultural factors in cancer care in india}

\section{Beliefs related to health and illness}

The beliefs in respect with health and illness vary across countries and regions. It is often difficult to distinguish between beliefs stemming from religious affiliation from those associated with ethnic or cultural background. ${ }^{16}$ Different religions have different views related to health, disease, illness and overall health care. In Hinduism, it is believed that cancer is due to past sins or karma. Besides religious beliefs, there are certain cultural beliefs also. In many traditional societies, disease is considered God's punishment, or one's Karma. ${ }^{17}$ In Western cultures, individuals are expected to be involved in an active struggle to "fight cancer" and regain health. In Eastern cultures, individuals are expected to live in harmony with nature, with passivity and fatalistic views being common. So in order to achieve common therapeutic goals, it remains really necessary that the patients and the caregivers both work through the contradictory views and beliefs that are related to cancer. ${ }^{18}$

\section{Religious and spiritual care}

Spiritual care plays a key role in cancer care. It is seen as an essential component of cancer care. It plays a prominent role especially in developing countries where medical and comfort resources are limited. Spiritual dimensions were reported to be most important aspects in the of quality of life in contrast to the functional aspects found in a study in an East Indian setting. ${ }^{19}$ Religion may be seen as a way of thinking about the spiritual issues that is prescribed by the group of people. ${ }^{20}$ It may shape approaches to spiritual care. Based on this, the caregivers In India employ different methods like prayer, chants, devotion, yoga and medication to improve their quality of life.

\section{Collusion}

Collusion is a very common phenomenon found in Indian settings where the family members often wish to know the truth about the cancer diagnosis but prefer to hide it from their ill family member. This tendency toward collusion does not persist across all cultural groups in India. In a study ${ }^{21}$ some subjects felt that sooner or later patients would find out about their disease, so facts should not be hidden from them. Collusion may be viewed as coming in the way of an individual's autonomy, and may deprive him of the benefits of quality care. Whenever collusion interferes with the care, it needs to be broken or handled. It is seen that the Indians are highly influenced by the principles of beneficence and non-male ficence. Families may want to protect patients by not discussing death and end-of-life decisions, which directly encourages collusion.

\section{Discussion}

The extensive literature review on the positive and negative psychological effects on the cancer caregivers clearly indicates that the caregiver's psychology suffers to a great deal due to the life consuming task of caregiving. As caregiving is a life changing task it leaves the caregivers stressed and depressed. One of the major psychological impacts that have emerged out of the literature review is depression and anxiety among the cancer caregivers. It leads to the deterioration of health and early deaths among the caregivers. So the identification of early symptoms of depression is a key factor in devising further intervention strategies for the caregivers. Once the depressive symptoms are caught in the caregivers, those having dysfunctional grief pattern can also be identified and prevented from reaching to clinical level of depression.

Besides depression, factors like patient's age and the stage of the illness have been found to be exerting great effect on the informal caregiver's psychological health. Also the caregiver's level of education and income seems to be a determining factor effecting the psychological health of the informal caregivers either in a positive or a negative way. Informal caregivers also derive positive utility and a high sense of self-esteem from the task of caregiving. But overall the negative psychological effects are much higher than a few positive ones. In the Indian context, certain cultural factors like the beliefs related to the concept of cancer, the religious and spiritual care and the act of collusion by the informal caregivers of cancer patients also play a determining role in caregiving. However whether these factors have a negative or positive effect on the psychology of caregivers, still needs to be probed.

\section{Implications and future directions}

A great deal of research has been done so far on the informal caregivers of cancer patients. However most of these studies have focused on the negative consequences and the grey side of caregiving. There is a great need to throw light on the positive impacts of caregiving on the psychology of caregivers. A study by Brouwer et al. ${ }^{15}$ came up with the findings of positive process utility derived from caregiving. Researches like this would change the mindset of the people from looking caregiving as a burdensome task to a satisfactory deed. More 
researches on the choice processes of becoming a caregiver needs to be done from different perspectives.

The health care systems depend heavily on the efforts of informal caregivers to assist their patients. So it is very important for the policymakers to realise the fact that certain policies need to be made for the informal caregivers in order to retain the benefits of informal care. In a traditional country like India certain belief systems that affect the psyche of the caregivers in a negative way, need to be evaded by intervention strategies. Also the benefits rendered by the spiritual and religious care should be propagated in order to maximise its positive effects and help the caregivers to give quality care without affecting them physically or psychologically. Financial care to alleviate the economic stress of informal caregivers, caregiver's education and support programmes along with the primary care interventions need to be addressed urgently.

It is highly recommended to know and address the unmet care needs of the informal cancer caregivers. In order to reduce the unpredictability caused by the diagnosis of the disease, it remains a matter of great concern to know the kind of information that the caregivers need in order to reduce their anxiety and stress. Health care providers must give the complete bunch of knowledge that is needed by the caregivers and hence support them in giving the quality care to the patients. Unfortunately the mode of helping only seems to be providing the knowledge and not the skills that are needed for its application, which further hampers the quality care. More researches are recommended to probe into the comparison between the caregivers and the non-caregivers in relation to certain outcome variables like social integration which will further enable more tailored interventions. Studies need to be done on how the various variable changes during the course of caregivers experience like social support, employment and strain. Very limited amount of research has been carried out to see how the caregiver's needs change with the changes in the patient's stage of illness. . Also in a developing country like India, it is warranted to provide specific attention to those caregivers who live only with the patient, with a low income, those with a distressed relationship and with a high level of patient dependency and care involvement.

We need to throw light on the number of caregivers in a particular population along with their characteristics and the arrangements for their care and support. Also we need to explore the kind of support the caregivers get from the health care services.

In India we can focus on the cultural factors that promote interdependence and compassion. The role of various cultural beliefs along with the effect of spiritual and religious care also needs to be taken into consideration. All these factors sum up to produce a healthy caregiving scenario. In the Indian context, we need to put efforts in order to mitigate the caregiving burden and improve the level of caregiving provided by the informal caregivers. This will greatly help the family unit. ${ }^{22}$ In order to promote the psychological health of the informal caregivers of cancer patients, there is a dire need to strengthen the family through counselling and various intervention plans. Government and society at large needs to come forward and join hands for this.

\section{Acknowledgments}

None.

\section{Conflicts of interest}

Author declares there are no conflicts of interest.

\section{Funding}

None.

\section{References}

1. Los Angeles Health. Informal caregiving: Implications for Public Health. Los Angeles, USA. 2010.

2. Kristjanson LJ, Aoun S. Palliative care for families: remembering the hidden patients. Can J Psychiatry. 2004;49(6):359-365.

3. Halm MA, Bakas T. Factors associated with caregiver depressive symptoms, outcomes and perceived physical health after coronary artery bypass surgery. J Cardiovasc Nurs. 2007;22(6):508-515.

4. World Health Organisation. World Cancer Report. Geneva, USA. 2015.

5. Sinha K. 1 million new cancer cases being diagnosed in India each year: Study. The Times of India. 2014.

6. Cotrim H, Pereira G. Impact of colorectal cancer on patient and family: Implications for care. Eur J Oncol Nurs. 2008;12(3):217-226.

7. Pinquart M, Sörensen S. Differences between caregivers and noncaregivers in psychological health and physical health: A meta-analysis. Psychol Aging . 2003;18(2):250-267.

8. Nijboer C, Triemstra M, Tempelaar R, et al. Determinants of caregiving experiences and mental health of partners of cancer patients. Cancer. 1998;86(4):577-588.

9. Grunfeld E, Coyle D, Whelan T, et al. Family caregiver burden: Results of a longitudinal study of breast cancer patients and their principal caregivers. CMAJ . 2004;170(12):1795-1801.

10. Northouse LL, Katapodi MC, Schafenacker AM, et al. The impact of caregiving on the psychological well-being of family caregivers and cancer patients. Semin Oncol Nurs. 2012;28(4):236-245.

11. Goldzweig G, Merims S, Ganon R, et al. Informal caregiving to older cancer patients: Preliminary research outcomes and implications. Ann Oncol . 2013;24(10):2635-2640.

12. Braun M, Mikulincer M, Rydall A, et al. Hidden morbidity in cancer spouse caregivers. J Clin Oncol. 2007;25(30):4829-4834.

13. Song JI, Shin DW, Choi JY, et al. Quality of life and mental health in family caregivers of patients with terminal cancer. Support Care Cancer. 2016;19(10):1519-1526.

14. National Centre on Caregiving. Family Caregiver Alliance. California, USA. 2014.

15. Brouwer WB, van Exel NJ, van den Berg B, et al. Process utility from providing informal care: The benefit of caring. Health Policy . 2004;74(1):85-99.

16. Miller MA. Culture, spirituality and women's health. J Obstet Gynecol Neonatal Nurs. 1995;24(3):257-263.

17. Chandra PS, Chaturvedi SK. Palliative care in India. Support Care Cancer. 1998;6(2):81-84.

18. Surbone A. Cultural aspects of communication in cancer care. Support Care Cancer. 2008;16(3):235-240.

19. Chaturvedi SK. Whats important to quality of life to Indians-in relation to cancer. Soc Sci Med. 1991;33(1):91-94.

20. Marstsolf DS. Cultural aspects of spirituality in cancer care. Semin Oncol Nurs. 1997;13(4):231-236.

21. Gautam S, Nijhavan M. Communicating with cancer patients. $B r J$ Psychiatry. 1987;150:760-764.

22. Avasthi A. Preserve and strengthen family to promote mental health. Indian J Psychiatry. 2010;52(2):113-126. 